ARTICLE

Received 4 May 2016 | Accepted 23 Nov 2016 | Published 14 Feb $2017 \quad$ DOl: 10.1038/ncomms14043 OPEN

\title{
A ligand-directed divergent catalytic approach to establish structural and functional scaffold diversity
}

\author{
Yen-Chun Lee ${ }^{1,2}$, Sumersing Patil ${ }^{1,2}$, Christopher Golz ${ }^{2}$, Carsten Strohmann², Slava Ziegler ${ }^{1}$, \\ Kamal Kumar ${ }^{1} \&$ Herbert Waldmann ${ }^{1,2}$
}

The selective transformation of different starting materials by different metal catalysts under individually optimized reaction conditions to structurally different intermediates and products is a powerful approach to generate diverse molecular scaffolds. In a more unified albeit synthetically challenging strategy, common starting materials would be exposed to a common metal catalysis, leading to a common intermediate and giving rise to different scaffolds by tuning the reactivity of the metal catalyst through different ligands. Herein we present a ligand-directed synthesis approach for the gold(I)-catalysed cycloisomerization of oxindolederived 1,6-enynes that affords distinct molecular scaffolds following different catalytic reaction pathways. Varying electronic properties and the steric demand of the gold(I) ligands steers the fate of a common intermediary gold carbene to selectively form spirooxindoles, quinolones or df-oxindoles. Investigation of a synthesized compound collection in cell-based assays delivers structurally novel, selective modulators of the Hedgehog and Wnt signalling pathways, autophagy and of cellular proliferation.

\footnotetext{
${ }^{1}$ Max-Planck-Institut für Molekulare Physiologie, Abteilung Chemische Biologie, Otto-Hahn-Straße 11, Dortmund 44227, Germany. ${ }^{2}$ Technische Universität Dortmund, Fakultät Chemie, Chemische Biologie, Otto-Hahn-Straße 6, Dortmund 44221, Germany. Correspondence and requests for materials should be addressed to K.K. (email: kamal.kumar@mpi-dortmund.mpg.de) or to H.W. (email: herbert.waldmann@mpi-dortmund.mpg.de).
} 
$\mathrm{n}$ the design and assembly of compound collections for chemical biology and medicinal chemistry research, the structural diversity of the core molecular scaffolds of the library members ${ }^{1,2}$ is decisive to guarantee high performance in biological investigations and to deliver structurally and functionally diverse small molecules ${ }^{3}$. Therefore, novel methods for the efficient synthesis of structurally distinct and diverse 'privileged' molecular scaffolds giving rise to compound classes with differing and selective bioactivity are in very high demand $^{4-6}$, yet remain among the most challenging problems putting organic synthesis itself at the heart of discovery in chemical biology and medicinal research ${ }^{7-9}$.

In established approaches for the synthesis of structurally different molecular scaffolds, usually starting materials are varied in structure and subjected to transformation by different reagents and catalysts, in particular metal catalysts under individually established reaction conditions. Depending on the structure of the individual starting material, the reaction conditions, the reagents and catalysts employed, structurally different intermediates and products may be formed ${ }^{10-12}$. In this approach, chemical diversity is established individually and step-wise via structural modification of the starting materials, the reagents and the catalyst in series of isolated experiments, which renders it laborious and time-consuming. An alternative and more unified approach would utilize common starting materials that when exposed to a common mode of metal catalysis lead to a common type of intermediate, whose molecular fate then is steered to yield structurally different scaffolds by tuning the reactivity of the metal catalyst through different ligands. We demonstrate that this unified ligand-directed divergent synthesis approach can provide facile and efficient access to structurally rich compound collections that in turn can provide small molecules with different and selective biological activity. Key to this approach is the identification of both efficient and flexible transformations that preferably proceed through one of the several possible intermediates of a given catalytic cycle, whose molecular fate can be directed into different reaction pathways leading to different molecular frameworks. We report that use of different ligands in gold(I)-catalysed transformations of oxindole-derived 1,6-enynes (1) steers the common gold carbene intermediate formed by 6-endo-dig cyclization to the selective formation of three distinct natural product-inspired scaffolds and via (Fig. 1) biological investigation of resulting structurally rich compound collection in cell-based assays reveals structurally novel, selective modulators of the Hedgehog (Hh) and Wnt signalling pathways, autophagy and of cellular proliferation.

\section{Results}

Design and optimization of catalytic divergent synthesis. Gold(I)-mediated cycloisomerization reactions of $1, n$-enynes $(n=5-7)$ are efficient molecular rearrangements that often pass through cyclopropyl gold carbene intermediates ${ }^{13-16}$, whose reactivity can be influenced by the properties of the ligands in the gold complex and by their substitution pattern ${ }^{17-19}$. Therefore, a wealth of gold and other coinage metal-catalysed transformations to generate distinct molecular scaffolds has been reported in recent years ${ }^{20-29}$. For gold(I)-catalysed transformations numerous different ligands, such as phosphines, phosphites, heterocyclic carbenes, halides and others are readily available. To explore whether the cyclopropyl gold carbene intermediates ${ }^{30-32}$ formed in the ene-yne cyclization could be directed into diverse reaction pathways by modulating the ligand structure and at the same time arriving at biologically relevant scaffolds ${ }^{33,34}$, skeletal rearrangements of oxindolederived 1,6-enynes (Fig. 1) were investigated. The oxindole scaffold is the structurally determining entity of a large class of natural products endowed with numerous bioactivities and has served as blueprint enabling the discovery of novel biologically active natural product-inspired compound classes ${ }^{35-38}$.

To explore the feasibility of the approach, oxindole-derived 1,6-enyne $(E: Z=3: 1)$ 1a was prepared from isatin by the addition of lithium phenylacetylide to the keto group and subsequent $O$-allylation of the resulting tertiary alcohol (Supplementary Methods). Treatment of the 1,6-enyne 1a with $5 \mathrm{~mol} \%$ of the cationic gold(I) complexes $\mathrm{Au}(\mathrm{OTf}) \mathrm{PPh}_{3}$ or $\mathrm{Au}\left(\mathrm{BF}_{4}\right) \mathrm{PPh}_{3}$ in dichloromethane (DCM) yielded spirooxindole $\mathbf{2 a}$ in low to moderate yield (entries 1-2, Table 1). In contrast, exposure of the enyne to $\mathrm{AuCl}_{3}$ under the same conditions led to the formation of structurally distinct cyclopropyl-substituted quinolone $\mathbf{3 a}$ in low yield (entry 3). Employment of a gold complex with an $N$-heterocyclic carbene bearing a highly electron donating ligand (I, entry 4), gave a mixture of $\mathbf{2 a}$ and 3a. Gratifyingly, treatment with the sterically demanding gold complexes II-IV in DCM selectively delivered 3a (entries 5-7, Table 1). Catalyst III yielded syn-quinolone $\mathbf{3} \mathbf{a}$ as major product in a preparatively viable yield of $67 \%$ (entry 6 , Table 1). The bulk of the biaryl moiety seems critical for the efficiency of the reaction since gold complexes II and IV embodying an unsubstituted biaryl ring $\left(\mathrm{R}^{1}=\mathrm{H}\right)$, but sterically equally or more demanding phosphines gave lower yields than complex III (entry 5,7).

We reasoned that employment of a more electrophilic gold complex like $\mathbf{V}$, employed by Nieto-Oberhuber et al. in $[4+2]$ cycloaddition reactions of 1,6 -enynes ${ }^{18}$ would favour migration

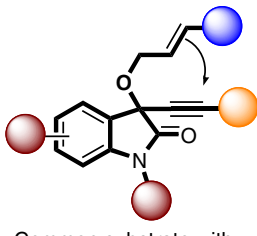

Common substrate with a privileged ring system

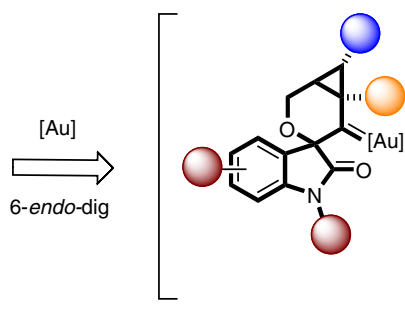

- Groups influencing reaction pathways an
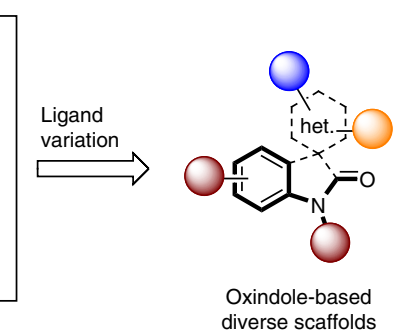

Sites for further modifications

Figure 1 | Design of ligand-directed gold catalysis approach to diverse scaffolds. The approach explores the potential of a common substrate, supporting a privileged oxindole ring system and carrying enyne functional groups that can be influenced differently to follow distinct reaction pathways and thereby generating distinct scaffolds. In particular, ligand variations in the catalytic gold complex can steer the common spirooxindole gold carbene intermediate, formed by gold(I)-mediated 6-endo-dig cycloisomerization, to different rearrangements and leading to diverse scaffolds. Het, heterocycle. 
Table 1 | Ligand-directed distinct cycloisomerizations of $\mathbf{O}$-crotylated substrate 1a to yield different scaffolds.
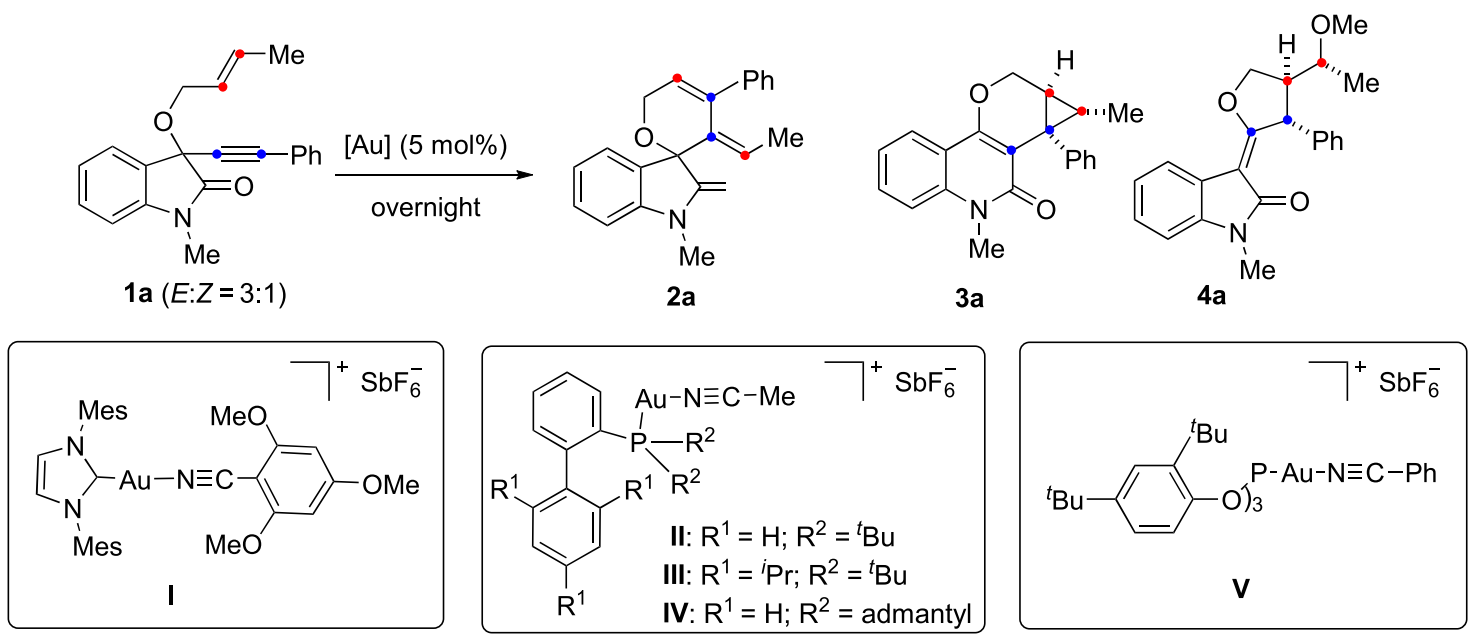

\begin{tabular}{|c|c|c|c|c|c|c|c|}
\hline \multirow[t]{2}{*}{ Entry } & \multirow[t]{2}{*}{ [Au] } & \multirow[t]{2}{*}{ Solvent } & \multirow[t]{2}{*}{ Nu. (eq) } & \multirow[t]{2}{*}{ Temp. } & \multicolumn{3}{|c|}{ Yield (\%)* } \\
\hline & & & & & $2 a$ & $3 a$ & $4 a$ \\
\hline 1 & $\mathrm{Au}(\mathrm{OTf}) \mathrm{PPh}_{3}$ & DCM & - & $r t$ & 20 & - & - \\
\hline 2 & $\mathrm{Au}\left(\mathrm{BF}_{4}\right) \mathrm{PPh}_{3}$ & DCM & - & rt & 33 & - & - \\
\hline $3^{\dagger}$ & $\mathrm{AuCl}_{3}$ & DCM & - & rt & - & 23 & - \\
\hline 4 & I & DCM & - & rt & 43 & 7 & - \\
\hline 5 & II & DCM & - & rt & - & 57 & - \\
\hline 6 & III & DCM & - & rt & - & 67 & - \\
\hline 7 & IV & DCM & - & rt & - & 43 & - \\
\hline 8 & $\mathbf{V}$ & DCM & - & rt & 60 & - & 一 \\
\hline 9 & $\mathbf{V}$ & DCE & $\mathrm{MeOH}(20.0)$ & $60^{\circ} \mathrm{C}$ & & & 20 \\
\hline 10 & II & DCE & $\mathrm{MeOH}(20.0)$ & $60^{\circ} \mathrm{C}$ & - & - & 62 \\
\hline 11 & III & DCE & $\mathrm{MeOH}(20.0)$ & $60^{\circ} \mathrm{C}$ & - & - & 56 \\
\hline 12 & IV & DCE & $\mathrm{MeOH}(20.0)$ & $60^{\circ} \mathrm{C}$ & - & - & 50 \\
\hline 13 & II & DCE & MeOH (10.0) & $60^{\circ} \mathrm{C}$ & - & - & 73 \\
\hline
\end{tabular}

of the cyclopropane ring conserved in 3a. Gratifyingly, $5 \mathrm{~mol} \%$ of gold complex $\mathbf{V}$ at room temperature in DCM led to the selective formation of spirooxindole $2 \mathrm{a}$ in $60 \%$ yield (entry 8 , Table 1 ).

To induce additional rearrangements of a plausible cyclopropane gold carbene intermediate, enyne 1a was exposed to catalysts II- $\mathbf{V}$ in the presence of methanol as nucleophile that might trigger cyclopropane ring opening. Indeed, treatment with catalyst $\mathbf{V}$ in dichloroethane (DCE) at $60^{\circ} \mathrm{C}$ yielded a novel product embodying the (E)-3-(dihydrofuran-2(3H)-ylidene) indolin-2-one scaffold ( $d f$-oxindole, entry 9 ) in low yield. Again, using the related gold complexes II-IV under the same reaction conditions revealed that a right balance of steric bias and electrophilic nature is the key to selectively form the $d f$-oxindoles (entries 9-12). Thus, catalyst II with bulky groups on the phosphine and unsubstituted biphenyl moiety afforded the $d f$-oxindole $\mathbf{4 a}$ in viable yield (entry 10 ), while catalysts III and IV with sterically demanding groups on the biphenyl moiety and the phosphine were less efficient (entries 11-12). Finally, reducing the amount of methanol gave the best yield of $d f$-oxindole $\mathbf{4 a}$ (73\%, entry 13, and Supplementary Table 1). The structures of products $\mathbf{2 a - 4 a}$ with different scaffolds were unambiguously ascertained by X-ray crystal structure analysis (Supplementary Tables 3-5).

These results demonstrate that variation of the ligand under otherwise nearly identical reaction conditions does allow to steer the fate of a most likely common cyclopropyl gold carbene intermediate (see below for a mechanistic rationale) into different trajectories giving rise to distinct and structurally diverse molecular scaffolds. The three scaffolds formed from the 1,6-enynes represent characteristic structural elements of different drug and natural product classes, and thus cover different areas of biologically relevant chemical space, raising the expectation that they may also yield novel modulators of different biological phenomena ${ }^{39-43}$.

Construction of a scaffold-rich compound collection. Variation of the oxindole substituents on the aryl ring, at the nitrogen and on the acetylene moiety allowed to rapidly synthesizing a small, but structurally diverse compound collection (Fig. 2). In all cases, the products were formed stereospecifically and in good to acceptable yields. In general, variation of electron density in the aryl ring of the oxindole and on the acetylene was well tolerated. 1,6-enynes with a thiophene as a representative heterocycle attached to the acetylene were also efficiently transformed into three different scaffolds (2e, $3 c$ and $4 e$, Fig. 2).

Mechanistic insights into gold-catalysed transformations. By analogy to previous observations for related gold-catalysed cyclorearrangements ${ }^{44-46}$, we assume that formation of scaffolds 2-4 plausibly occurs via a common spirooxindole gold carbene 6 formed by 6-endo-dig cyclization of enynes 1 (Fig. 3). Of the three 


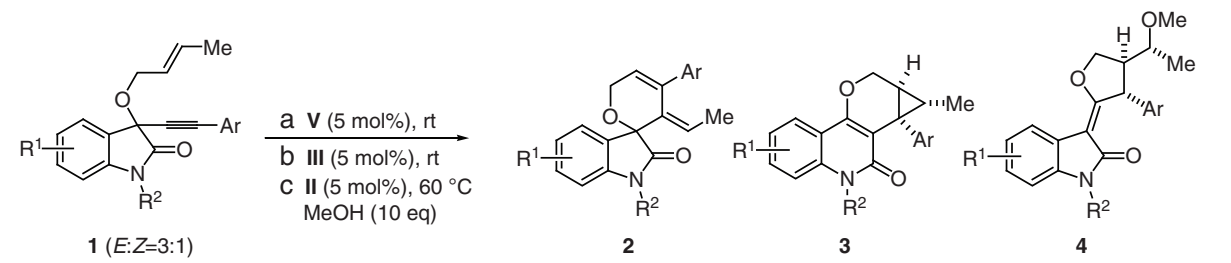

\section{a}

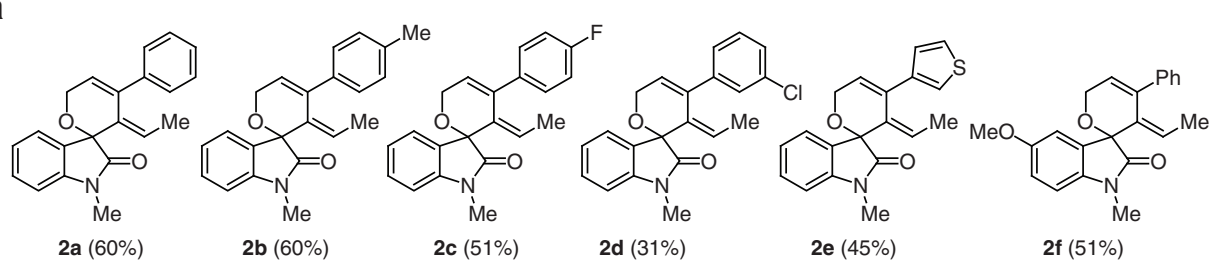<smiles>COC(=O)C1=C(c2ccccc2)C=CCO1</smiles>

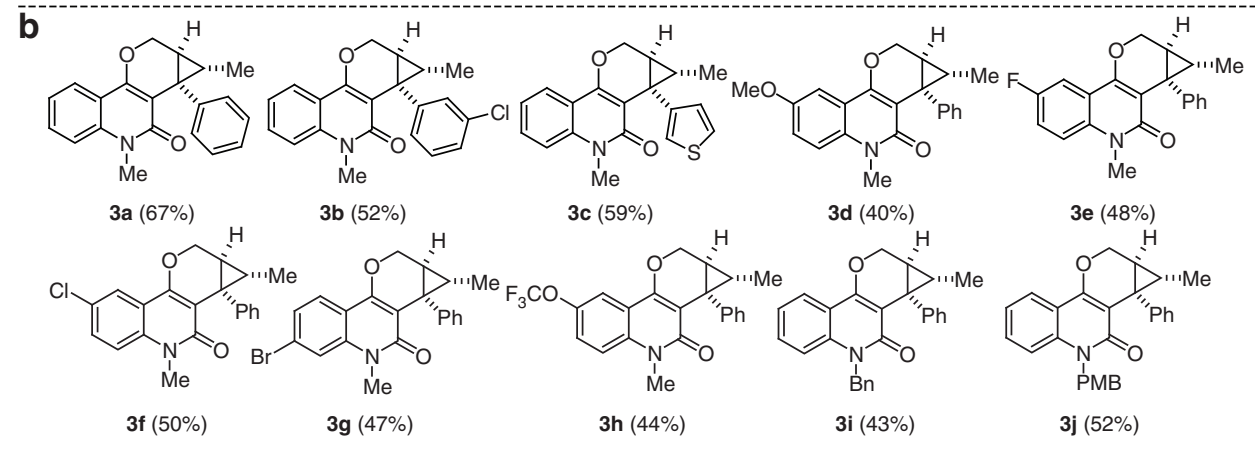

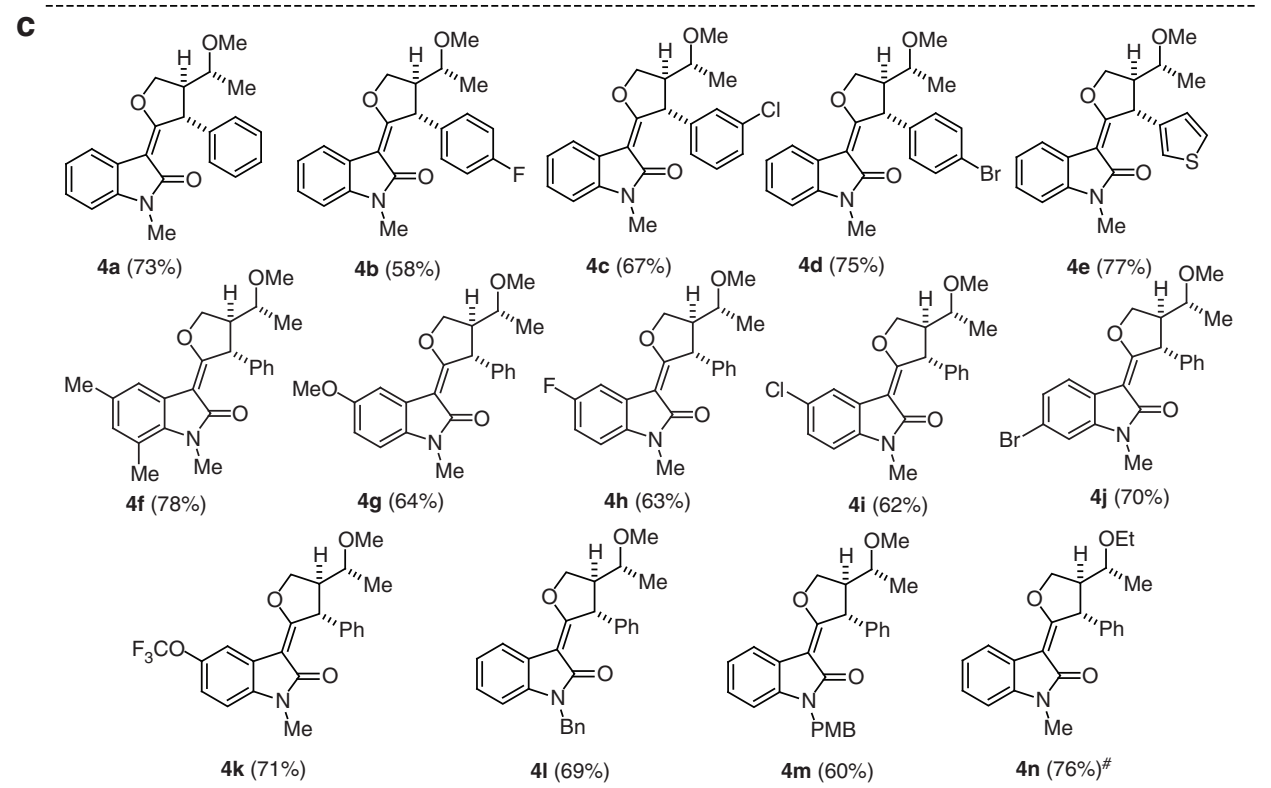

\# EtOH was used as nucleophile

Figure 2 | A collection of diverse small molecules obtained via LDS appraoch. (a) Synthesis of spirooxindoles 2: 1,6-enynes 1 (0.1 M), $\mathbf{V}(0.5$ mol\%), $\mathrm{DCM}$ and $0^{\circ} \mathrm{C}$ to $\mathrm{rt}$, overnight. (b) Synthesis of quinolones 3: 1,6-enynes $\mathbf{1}(0.1 \mathrm{M})$, III $(0.5 \mathrm{~mol} \%)$ and $\mathrm{DCM}, 0^{\circ} \mathrm{C}$ to rt, overnight. (c) Synthesis of df-oxindoles 4: 1,6-enynes 1 (0.1 M), MeOH (10 eq), II (0.5 mol\%), DCE and rt to $60^{\circ} \mathrm{C}$, overnight. Bn, benzyl; DCE, dichloroethane; eq, equivalent; LDS, ligand-directed divergent synthesis; PMB, $p$-phenoxy benzyl; rt, room temperature. 
a
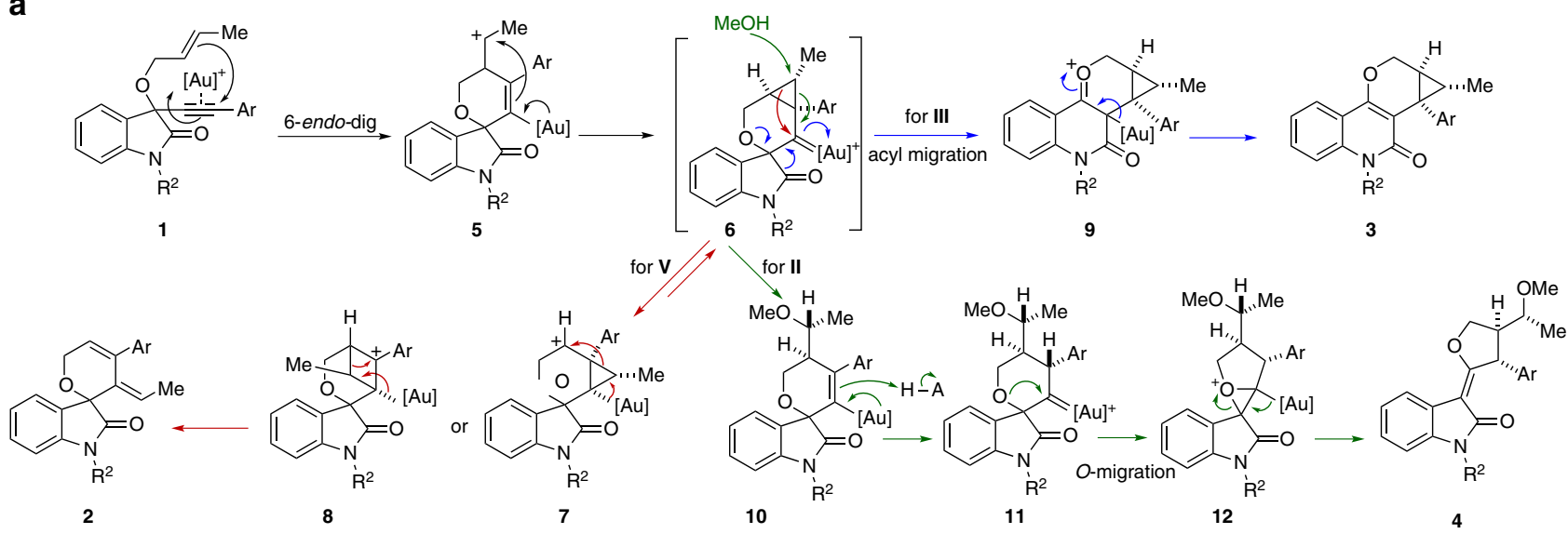

b

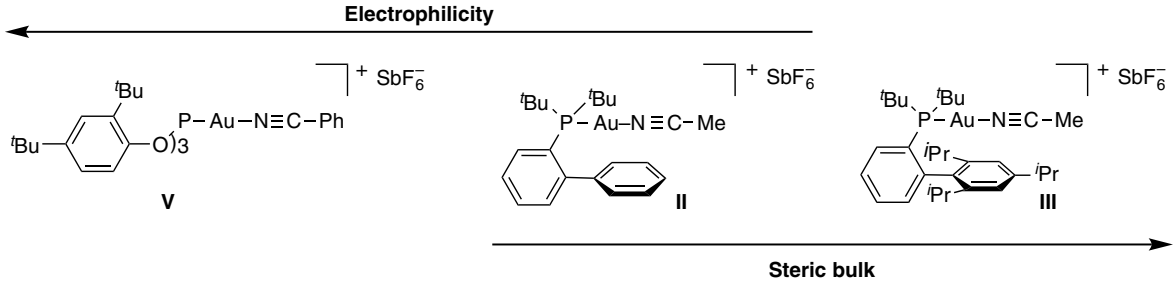

Figure 3 | Proposed mechanistic pathways of divergent gold(I) catalysis to afford different scaffolds. (a) The spirooxindole gold carbene intermediate 6 was formed by the cationic gold(I) activation of alkyne 1, followed by 6-endo-dig cyclization and cyclopropane formation. The most electrophilic gold(I) catalyst $\mathbf{V}$ induces a sequential cyclopropane migration and deauration to generate spirooxindole $\mathbf{2}$ (magenta arrows). Very bulky gold complex III promotes the pinacol type acyl migration followed by deauration to afford quinolone $\mathbf{3}$ (blue arrows). In the presence of $\mathrm{MeOH}$, the less bulky but relatively more electrophilic gold complex II allows the 1,4-nucleophilic addition of methanol to open up the cyclopropane ring (10). Subsequent protonation, spiroether insertion and deauration in $\mathbf{1 0}$ generate the $d f$-oxindole $\mathbf{4}$ (turquoise arrows). (b) A comparison of the molecular properties of the cationic gold catalysts II, III and $\mathbf{V}$.

catalysts yielding the products with different scaffolds with highest yields, gold complex $\mathbf{V}$ is most electrophilic. In the presence of this catalyst, cyclopropane ring migration may be favoured to yield rearrangement product 7 or the more stable benzylic carbocation $\mathbf{8}$ that ultimately will lead to the spirooxindole 2 (magenta arrows, Fig. 3). We furthermore assume that in the presence of the very bulky gold complex III, intermediate 6 tends to relieve the strain of the spiro-ring by means of a pinacol type acyl migration thereby forming oxonium-quinolone gold intermediate 9. Subsequent deauration leads to the formation of tetracyclic quinolone 3 (blue arrows, Fig. 3). In the presence of the relatively less bulky but more electrophilic gold complex II, intermediate 6 will be relatively more stabilized to favour nucleophilic addition with cyclopropane ring opening (10) followed by protonation to yield anti-gold carbene intermediate $\mathbf{1 1}$ (see also 18 in Fig. 5a). Intramolecular nucleophilic attack by the spiroether will lead to highly strained and reactive tetracyclic oxonium ion $\mathbf{1 2}$ that by deauration and concomitant ring opening will give rise to $d$-oxindole 4 (turquoise arrows, Fig. 3$)^{47}$.

In all rearrangements of 1,6-enynes 1 (Fig. 3), the substitution pattern on the olefin will have a major influence on the stability of carbocation intermediate $\mathbf{5}$ and susceptibility of the cyclopropane ring in intermediate $\mathbf{6}$ to nucleophilic attack. Thus, we reasoned that increasing the stability of the intermediary carbocation might open paths to different rearrangements and scaffolds. To explore this notion, dimethyl substituted enynes 13 were exposed to different gold complexes, and gratifyingly treatment with highly electrophilic gold complex II in DCM selectively yielded structurally different $d f$-oxindoles 14 . Solvent variation (Supplementary Table 2) revealed that in tetrahydrofuran product 14a was formed in almost quantitative yield as a single diastereoisomer (Fig. 4).
Synthesis of $d f$-oxindoles from $O$-prenylated substrates. Formation of $d f$-oxindoles 14 occurs by an analogous initial 6-endo-dig cyclization of the olefin to the acetylene activated by gold complex II to form cyclopropane gold carbene 15 (see Fig. 5a for conversion of 13 into 14). However the gem-dimethyl substitution facilitates cyclopropane ring opening via relatively stable carbocation $\mathbf{1 6}$ and proton loss to yield intermediate $\mathbf{1 7}$. Protonation yields anti-gold carbene intermediate $\mathbf{1 8}$ that sets the stage for the formation of analogous oxiranium ion 19 and final rearrangement to unsaturated $d f$-oxindoles 14 (Fig. 5). This mechanistic proposal is supported by deuterium incorporation and formation of the corresponding deutero-methyl ether in the presence of $\mathrm{CD}_{3} \mathrm{OD}$ (Fig. $5 \mathrm{~b}$ ). The structure of $d f$-oxindole 14a was unambiguously ascertained by X-ray crystal structure analysis (Supplementary Table 6).

The rearrangement tolerates different substituents on the indole and the acetylenic moiety, including thiophene as representative heterocycle $(\mathbf{1 4 g})$ and affords products 14 as single anti-diastereoisomers (Fig. 4). Alkyl-substituted acetylenes exhibit slower conversion, but in the presence of $10 \mathrm{~mol} \%$ catalyst the corresponding $d f$-oxindoles $(\mathbf{1 4 h}-\mathbf{i})$ were formed stereoselectively and in good yields. By analogy variation of the substituents on the aryl part of the oxindole and of the $N$-protecting group yielded the corresponding $d f$-oxindoles $(\mathbf{1 4} \mathbf{j}-\mathbf{p})$ and $(\mathbf{1 4 q}-\mathbf{u})$, respectively, in good to excellent yields as single diastereoisomers.

Discovery of small molecules with orthogonal biological activities. To explore if the scaffold diversity in the compound collection translates into selective biological activity, the synthesized compound collection was exposed to cellular assays monitoring different biological phenomena. Much to our delight we identified individual compounds that are structurally novel 


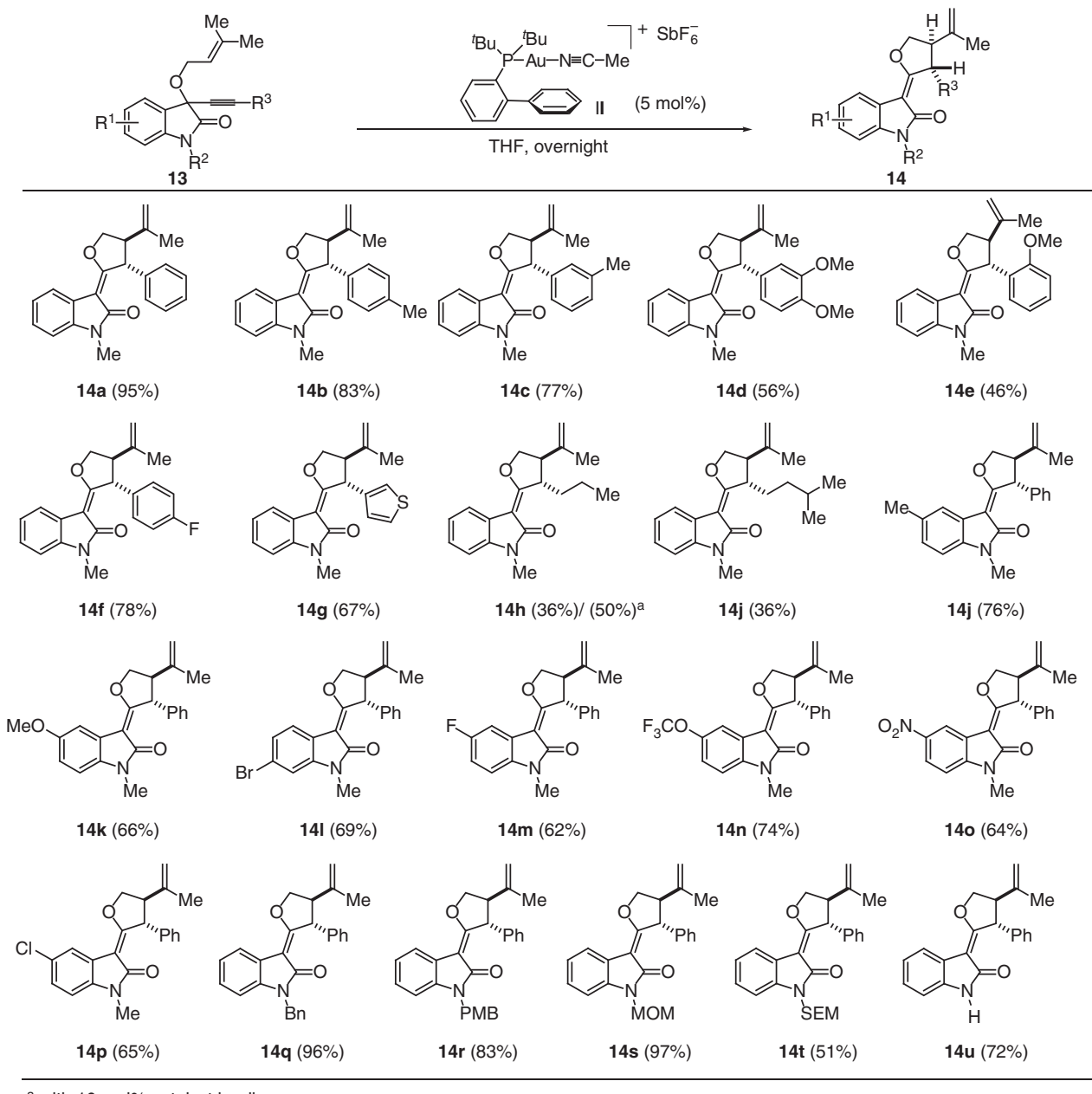

a with $10 \mathrm{~mol} \%$ catalyst loading.

Figure 4 | Scope of the gold catalysed rearrangement of $\boldsymbol{O}$-prenylated substrate $\mathbf{1 3}$ to $\mathbf{d f}$-oxindoles $\mathbf{1 4}$. Reaction condition: 1,6-enynes $\mathbf{1 3}$ (0.1 M), II $(0.5 \mathrm{~mol} \%), \mathrm{THF}, 0^{\circ} \mathrm{C}$ to $\mathrm{rt}$, overnight. MOM, methoxymethyl; SEM, (2-(trimethylsilyl)ethoxy)methyl; THF, tetrahydrofuran.

selective inhibitors of the $\mathrm{Hh}$ and Wnt signalling pathways, autophagy and of cellular proliferation and displayed largely orthogonal biological activity.

The Hh pathway is an evolutionarily conserved developmental signalling pathway that governs vital processes such as cell proliferation, differentiation, body patterning, tissue repair and regeneration ${ }^{48}$. In the absence of ligand, the membrane receptor Patched represses the seven-transmembrane protein Smoothened (Smo). The Hh pathway is activated on binding of the Hh ligand to Patched-1, thereby relieving the inhibition of Smo. Through a series of events that occur in the primary cilium, Smo promotes activation of Gli transcription factors that drives the transcription of Hh target genes. Aberrations in Hh signalling are associated with birth defects and cancer, including medulloblastoma and basal cell carcinoma ${ }^{48}$. Therefore, development of small-molecule modulators of Hh pathway is of utmost importance in cancer research.

Hh pathway inhibitors were identified by means of an osteogenesis assay that monitors $\mathrm{Hh}$ signalling activity in pluripotent mouse mesenchymal C3H10T1/2 cells on stimulation with purmorphamine ${ }^{49}$. Several oxindoles inhibited osteogenesis with half-maximal inhibitory concentrations $\left(\mathrm{IC}_{50}\right)$ in the low micromolar range, as detected by reduced activity of the osteogenic marker alkaline phosphatase.

The most potent compounds $\mathbf{1 4 q}\left(\mathrm{IC}_{50}=2.8 \mu \mathrm{M}\right)$ and $\mathbf{1 4 r}$ $\left(\mathrm{IC}_{50}=3.1 \mu \mathrm{M}\right.$; Fig. $6 \mathrm{a}$; Supplementary Table 7$)$ were additionally characterized in the orthogonal Gli reporter gene assay using Shh
Light II cells, a NIH/3T3-derived cell line that is stably transfected with Gli-responsive firefly luciferase and constitutive Renilla luciferase reporters ${ }^{50}$. 14q and $14 \mathbf{r}$ inhibited the Gli-dependent luciferase activity with $\mathrm{IC}_{50}$ values of 1.7 and $0.8 \mu \mathrm{M}$, respectively (Fig. 6b). Both compounds did not affect the viability of C3H10T1/2 cells and Renilla luciferase activity in Shh Light II cells, therefore, suggesting a specific inhibition of Hh signalling.

To further confirm and characterize Hh pathway inhibition, we monitored the expression of the Hh target genes Ptch1 and Gli1 on compound treatment ${ }^{48}$. Ptch1 is a negative regulator and Gli1, a positive regulator of $\mathrm{Hh}$ signalling, and both control the feedback regulation of the pathway. On treatment of $\mathrm{C} 3 \mathrm{H} 10 \mathrm{~T} 1 / 2$ cells with the compounds together with purmorphamine the expression of Ptch1 and Gli1 was suppressed in a dose-dependent manner (Fig. 6c,d).

Several small molecules like vismodegib and cyclopamine that modulate Hh signalling target the seven-transmembrane receptor $\mathrm{Smo}^{48}$. Thus, we assessed the ability of $14 \mathbf{q}$ and $14 \mathbf{r}$ to bind to Smo by means of competition experiments employing boron-dipyrromethene (BODIPY)-cyclopamine in HEK293T cells ectopically expressing $\mathrm{Smo}^{50}$. 14q and $\mathbf{1 4 r}$ could displace BODIPY-cyclopamine as observed by the decrease in BODIPY fluorescence (Fig. 7a). Concentration-dependent displacement of BODIPY-cyclopamine by $14 q$ and $14 \mathbf{r}$ was detected using flow cytometry (Fig. 7b), suggesting that 14q and 14r most likely inhibit the Hh pathway by directly binding to Smo. 
a

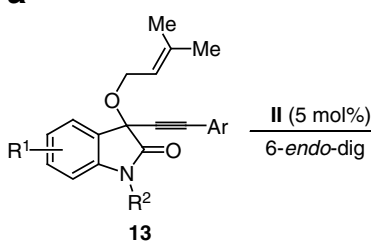

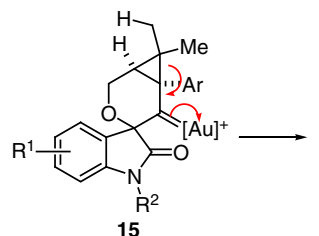

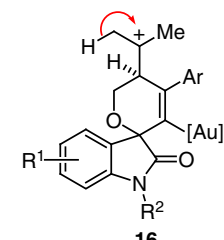

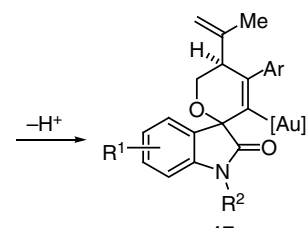

16

17

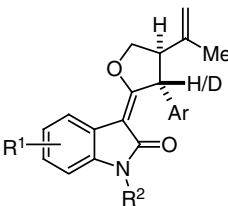<smiles>C1CCCCC1</smiles>

14

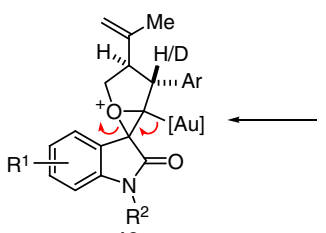

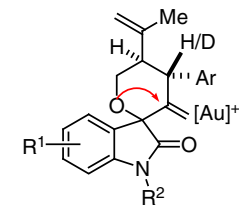

18

b<smiles></smiles>

13<smiles>C=C(C)[C@H]1CO/C(=C2/C(=O)N(C)c3ccccc32)[C@@]1(O)c1ccccc1</smiles>

14 (D 40\%) $(12 \%)$

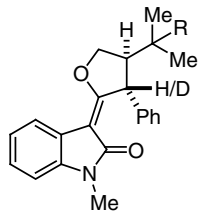

$20\left(\mathrm{R}=\mathrm{OCD}_{3}, \mathrm{D} 50 \%\right)$

Figure 5 | Mechanistic proposal for the formation of $\boldsymbol{d f}$-oxindole 14. (a) The proposed mechanism of the formation of $d f$-oxindole $\mathbf{1 4}$ involves the gold(I)-mediated 6-endo-dig cycloisomerization of 1,6-enyne to afford $\mathbf{1 5}$, followed by subsequent deprotonation, protonation, formation of a strained tetracyclic spiro-oxenium intermediate (19) and its rearrangement to afford 14. (b) In the presence of $d-\mathrm{MeOH}$ (as nucleophile as well as deuterium source), $d f$-oxindole $\mathbf{1 4}$ and $d-\mathrm{MeOH}$ adduct $\mathbf{2 0}$ of $d f$-oxindole was obtained in $14 \%$ yield with $40 \%$ deuteration at benzylic position and $47 \%$ yield with $50 \%$ deuteration at benzylic position, respectively. $\mathrm{MeOH}-d_{4}$, deuterated methanol.

a<smiles>C=C(C)[C@H]1CO/C(=C2/C(=O)N(C(C)(C)C)c3ccccc32)[C@H]1c1ccccc1</smiles>

$14 \mathrm{r}$<smiles>C=C(C)[C@H]1CO/C(=C2/C(=O)N(Cc3ccccc3)c3ccccc32)[C@H]1c1ccccc1</smiles>

$14 q$ b

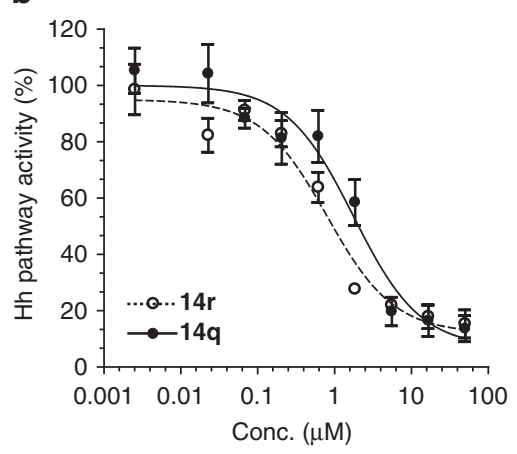

C

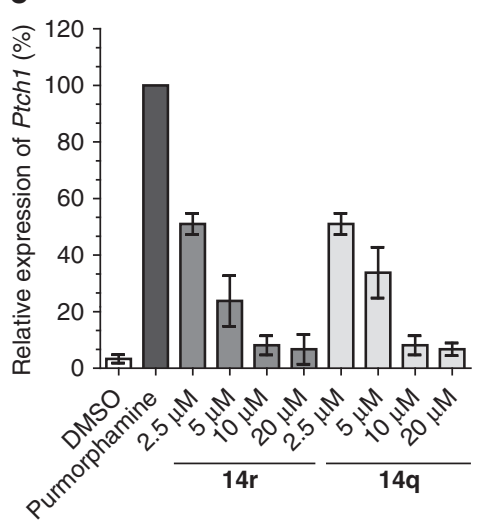

d

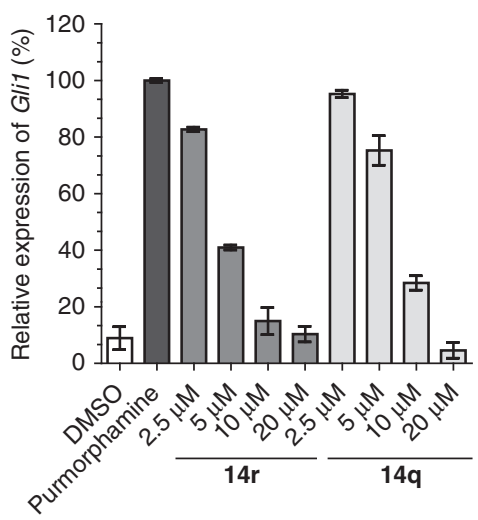

Figure 6 | 14q and 14r inhibit Hh signalling. (a) Structures of $\mathbf{1 4} \mathbf{r}$ and $\mathbf{1 4 q}$. (b) 14r and 14q inhibit Gli-dependent reporter gene expression in Shh Light II cells. Cells were treated with $1.5 \mu \mathrm{M}$ purmorphamine and different concentrations of compounds for $48 \mathrm{~h}$. Firefly and Renilla luciferase activities were then determined and ratios of firefly luciferase/Renilla luciferase signals were calculated, which are a measure of Hh pathway activity. Nonlinear regression analysis was performed using a four parameter fit. 14r and 14q suppress the expression of the Hh target genes Ptch1 (c) and Gli1 (d). C3H10T1/2 cells were treated with purmorphamine $(1.5 \mu \mathrm{M})$ and different concentrations of $\mathbf{1 4 r}$ and $\mathbf{1 4 q}$ or DMSO as a control for $48 \mathrm{~h}$ before isolation of total RNA. Following complementary DNA (cDNA) preparation, the relative expression levels of Ptch1, Gli1 and Gapdh were determined by means of quantitative PCR employing specific oligonucleotides for Ptch1 and Gli1 or Gapdh as a reference gene. Expression levels of Ptch1 and Gli1 were normalized to the levels of Gapdh and are depicted as percentage of gene expression in cells activated with purmorphamine (100\%). All data are mean values of three independent experiments $(n=3) \pm$ s.d. 
a

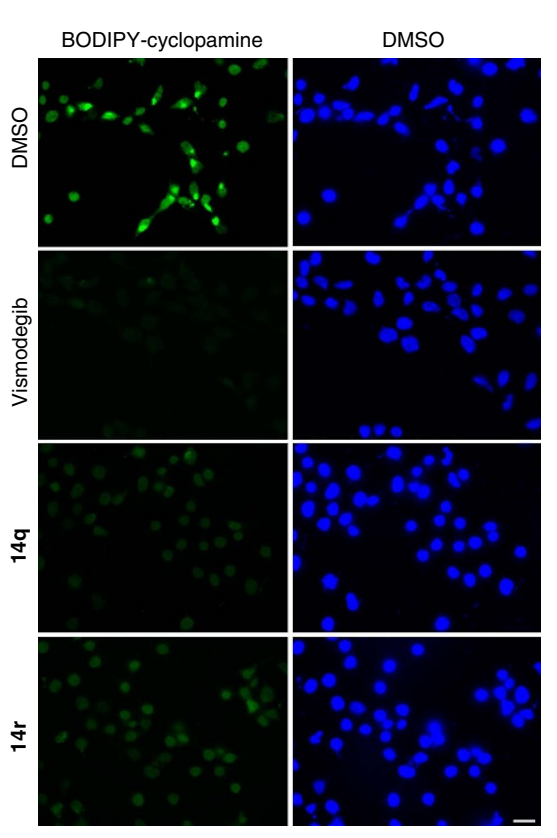

b

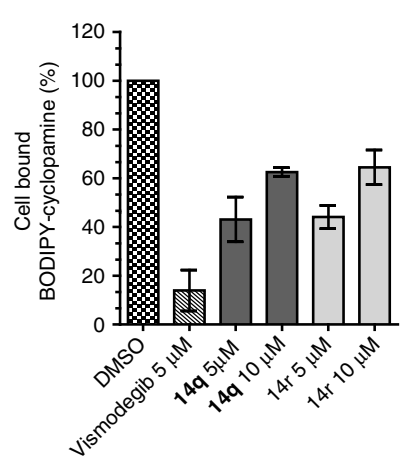

C

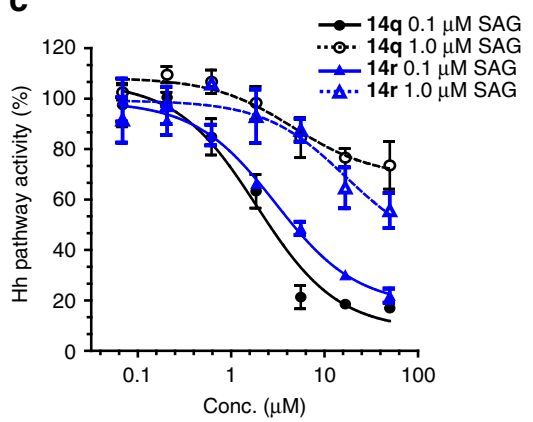

d
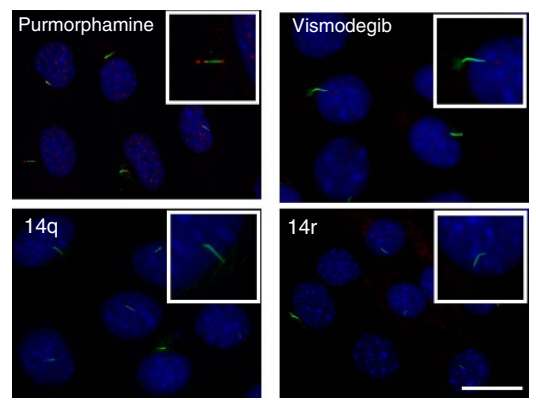

Figure 7 | 14q and 14r inhibit Smo. (a) 14q and 14r displace BODIPY-cyclopamine from Smo. HEK293T cells were transiently transfected with Smo expressing plasmid or empty vector. Forty-eight hours later cells were treated with BODIPY-cyclopamine $(5 \mathrm{nM}$, green) followed by addition of $10 \mu \mathrm{M}$ of 14q or $\mathbf{1 4} \mathbf{r}$ or vismodegib $(5 \mu \mathrm{M})$ and DMSO as controls. Cells were incubated for $1 \mathrm{~h}$ before fixation and staining with DAPI (4',6-diamidino-2phenylindole) to visualize the nuclei (blue). Scale bar, $20 \mu \mathrm{m}$. (b) HEK 293T cells ectopically expressing Smo were treated with different concentration of the compounds (14q and $\mathbf{1 4}$ ) , vismodegib or DMSO as controls in the presence of BODIPY-cyclopamine ( $5 \mathrm{nM})$ for $5 \mathrm{~h}$. The graph shows the percentage of cell-bound BODIPY-cyclopamine as detected by fluorescence-activated cell sorting analysis. Data are mean values of three independent experiments $(n=3) \pm$ s.d. (c) Influence of $\mathbf{1 4 q}$ and $\mathbf{1 4} \mathbf{r}$ on the Gli-mediated reporter gene expression on Hh pathway activation in Shh Light II cells by SAG (0.1 and $1 \mu \mathrm{M})$. Nonlinear regression analysis was performed using a four parameter fit. Data are mean values of three independent experiments $(n=3) \pm \mathrm{s} . \mathrm{d}$. and were normalized to cells treated with the respective concentration of SAG (set to $100 \%$ ). (d) NIH/3T3 cells were treated with purmorphamine (1.5 $\mu$ M) for $2 \mathrm{~h}$ followed by addition of vismodegib $(2 \mu \mathrm{M}), \mathbf{1 4 q}(5 \mu \mathrm{M})$ and $\mathbf{1 4} \mathbf{r}(5 \mu \mathrm{M})$, and further incubation for $12 \mathrm{~h}$. Cells were then fixed and stained to visualize the nuclei (DAPI, 4',6-diamidino-2-phenylindole; blue), Smo (red) and cilia (acetylated tubulin; green). Inset: representative single cilia. Scale bar, $10 \mu \mathrm{m}$.

To further characterize inhibition of the Hh pathway by $\mathbf{1 4 q}$ and 14r, we activated the pathway with the Smo agonist SAG. High concentrations of SAG saturate the binding sites on Smo, resulting in decreased inhibitory activity of Smo antagonists (for example, vismodegib), whereas the action of Hh pathway inhibitors that act downstream of Smo (for example, GANT61) is not impaired (Supplementary Fig. 1$)^{51}$. Indeed, the ability of $\mathbf{1 4 q}$ and $14 \mathbf{r}$ to inhibit the Gli-responsive reporter gene expression was reduced, when $1 \mu \mathrm{M}$ SAG was used instead of $0.1 \mu \mathrm{M}$ SAG (Fig. 7c), providing further proof that $\mathbf{1 4 q}$ and $\mathbf{1 4 r}$ inhibit $\mathrm{Hh}$ signalling by binding to Smo.

In vertebrate $\mathrm{Hh}$ signalling, Patched-1-induced inhibition of Smo is relieved on binding of the Hh ligand to Patched-1. Most of the Hh signalling cascade events occur in the primary cilium and involve translocation of multiple pathway components into this sensory organelle ${ }^{48}$. On $\mathrm{Hh}$ pathway activation Smo is laterally trafficked to the primary cilium via intraflagellar transport by binding to the kinesin-like protein KIF3a. However, genetic studies demonstrated that Smo is constantly cycling through the cilium without activating the pathway. Thus, localization of Smo into the cilium might be necessary, but not sufficient for $\mathrm{Hh}$ pathway activity ${ }^{52}$. To investigate the influence of the compounds on Smo trafficking to the primary cilium, we visualized Smo and acetylated tubulin as a ciliary marker. Purmorphamine promotes the localization of Smo in the primary cilium (Fig. 7d). However, on treatment with $\mathbf{1 4 q}$ and $\mathbf{1 4 r}$, the purmorphamine-induced ciliary localization of Smo is prevented similarly to the treatment with the Smo antagonist vismodegib (Fig. 7d).

To rule out any covalent binding of $d f$-oxindoles causing inhibition of Hh signalling, a wash out step was included $30 \mathrm{~min}$ post addition of the compounds in the Gli reporter gene assay and the BODIPY-cyclopamine displacement experiment. Removal of the compounds almost completely abolished their activity to suppress the expression of the reporter gene (Supplementary Fig. 2a) and to displace BODIPY-cyclopamine (Supplementary Fig. 2b). Thus, most likely 14q and 14r reversibly modulated Hh signalling.

As example for a different major cellular signalling cascade, we analysed whether the compound collection contains selective inhibitors of the Wnt signalling pathway. Wnt signalling is involved in the regulation of cell proliferation, migration and polarity, tissue regeneration and stem cell renewal, and is a major pathway with relevance to the establishment of cancer ${ }^{53}$. Wnt signalling modulators are widely employed to dissect signal progression through the pathway ${ }^{53}$. In canonical Wnt signalling, the absence of a Wnt signal results in a low protein level of the central player $\beta$-catenin. In the nucleus, transcription factors of the T-cell factor/lymphoid enhancer factor (TCF/LEF) family inhibit transcription of Wnt target genes by recruiting histone deacetylases. On Wnt activation, $\beta$-catenin accumulates in the cytoplasm and translocates to the nucleus, where it associates with TCF/LEF and recruits transcriptional coactivators and 
a

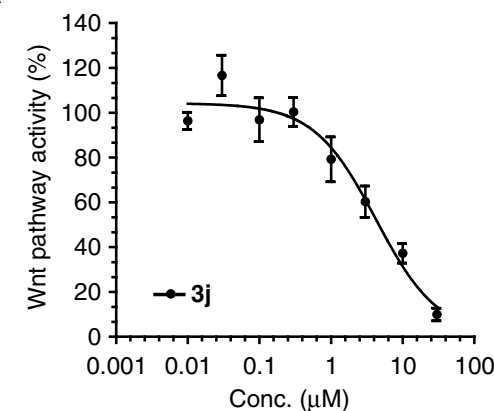

b

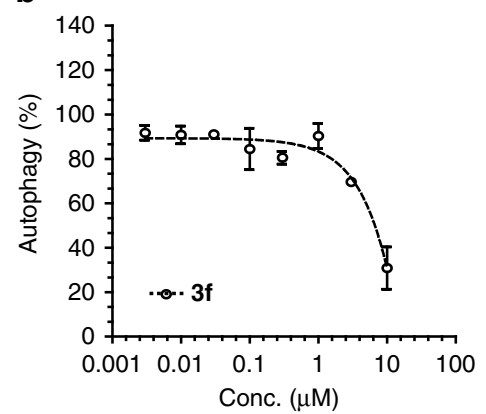

C

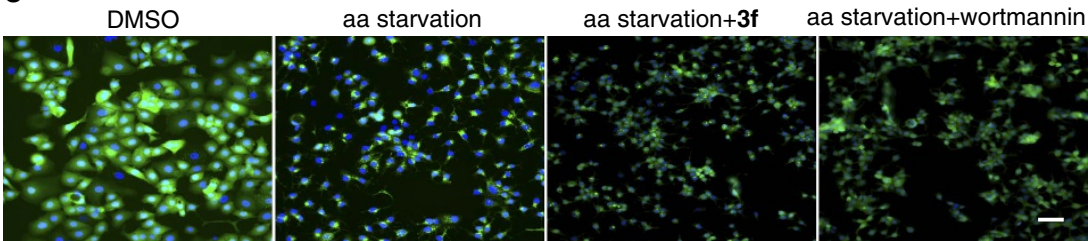

Figure 8 | Influence of representative compounds on Wnt signalling (3j) and autophagy (3f). (a) Dose-dependent inhibition of the Wnt pathway as determined by means of Wnt reporter gene. HEK293 cells stably transfected with the human Frizzled-1 receptor and a TOPFLASH-driven luciferase reporter gene were treated with different concentrations of $\mathbf{3 j}$ for $6 \mathrm{~h}$. Expression of the firefly luciferase as a reporter gene was the determined by means of luminescence as readout. Nonlinear regression analysis was performed using a four parameter fit. Data are mean values of three independent experiments $(n=3) \pm$ s.d. (b) Dose-response curve for inhibition of autophagy by 3f. MCF7-GFP-LC3 cells were deprived of amino acids to induce autophagy and treated with the different concentration of $\mathbf{3 f}$ for $3 \mathrm{~h}$. GFP-LC3 was detected as a measure of autophagosome formation. Nonlinear regression analysis was performed using a four parameter fit. Data are mean values of three independent experiments $(n=3) \pm$ s.d. (c) MCF7 cells that stably express GFP-LC3 (green) were starved for amino acids (aa) in the presence of the compound $\mathbf{3 f}(10 \mu \mathrm{M})$ or Wortmannin $(3 \mu \mathrm{M})$ for $3 \mathrm{~h}$ before fixation and staining of the DNA using Hoechst 33342 (blue). Autophagy induction is detected as an accumulation of GFP-LC3 puncta on starvation. Scale bar, $10 \mu \mathrm{m}$.

chromatin remodelling complexes to initiate the expression of Wnt target genes. In many epithelial cancers, the Wnt pathway is constitutively active as a result of mutations in different components of the pathway.

We screened the compound collection for modulation of Wnt signalling using a HEK293 reporter cell line that was stably transfected with the human Frizzled-1 receptor and a TOPFLASH-driven luciferase reporter gene ${ }^{54}$. Dose-response analyses were carried out for hit compounds for which cell viability remained $>80 \%$ with respect to control experiments. To rule out any direct inhibition of firefly luciferase or interference with transcription or translation, hit compounds were assayed for modulation of luciferase in HEK293 cells with constitutive luciferase expression. Pleasingly, the compound collection revealed hits belonging to the quinolone sub-library. For instance compound $\mathbf{3 j}$ (Fig. $8 \mathrm{a}, \mathrm{IC}_{50}=4.2 \mu \mathrm{M}$ ) is a structurally novel Wnt pathway inhibitor, which does not interfere with $\mathrm{Hh}$ signalling (Supplementary Table 7).

The compound collection was further assessed for modulation of autophagy. Autophagy is a catabolic self-digestive process that is important for maintaining cell homoeostasis through degradation of cellular components, for example, unfolded proteins and damaged organelles like mitochondria, ribosomes and the endoplasmic reticulum, which ensures their regular turnover ${ }^{55}$. Autophagy protects cells under stress conditions, is regarded as a survival mechanism and may provide energy and metabolites to cancer cells that usually are under metabolic stress for the lack of oxygen and nutrients and facing an increased energy demand. Thus, inhibition of autophagy is a promising strategy for targeting tumour cells or sensitizing cancer cells to anti-cancer therapies ${ }^{56}$. The compound collection was screened for autophagy modulation in MCF7 cells that stably express the autophagosome marker LC3 coupled to green fluorescent protein (GFP) to monitor accumulation of GFP-LC3-II on induction of autophagy by amino-acid starvation.
The phenotypic assay revealed that several quinolone and $d f$-oxindole derivatives dose-dependently inhibited autophagy with $\mathrm{IC}_{50}$ values in the low micromolar range. The quinolone $3 \mathrm{f}$ was the most effective molecule in modulating autophagy with an approximate $\mathrm{IC}_{50}$ value of $4.8 \mu \mathrm{M}$ (Fig. $8 \mathrm{~b}, \mathrm{c}$ ).

Notably, quinolone $3 \mathbf{f}$ displayed no activity in the $\mathrm{Hh}$ and the Wnt signalling assays, and Hh pathway inhibitors $\mathbf{1 4 q}$ and $\mathbf{1 4 r}$ and Wnt signalling inhibitor $\mathbf{3 j}$ did not influence autophagy (Supplementary Table 7).

Finally, we could identify modulators of cell proliferation by means of time-lapse imaging using an IncuCyte ZOOM device, monitoring cell confluency. The assay revealed spirooxindolebearing compounds as promising class of bioactive compounds affecting proliferation in HeLa cells. Spirooxindole $2 \mathbf{d}$ reduced the proliferation of $\mathrm{HeLa}$ cells with an $\mathrm{IC}_{50}$ of $15.4 \mu \mathrm{M}$ (Supplementary Fig. 3a,b; Supplementary Movies 1 and 2), but displayed no activity in the Hh and Wnt signalling assay and in modulation of autophagy. Vice versa, autophagy inhibiting quinolone 3f, Hh signalling inhibitors $\mathbf{1 4 q}$ and $\mathbf{1 4 r}$ and Wnt pathway inhibitor $\mathbf{3 j}$ did not reduce cell proliferation at comparable concentrations and thus are selective inhibitors of the respective pathway/process (Supplementary Table 7).

Although the scaffolds 2, 3, 4 and 14 do not hold reactive Michael acceptor functionalities often responsible for covalent binding to cellular targets under mild acidic conditions, nucleophilic addition to olefins might occur. To address this concern, stability of the compounds in the presence of glutathione (GSH) was determined for each scaffold (2d, 3f, 3j, 4i, 14q-r) and after different incubation times $(1,24$ and $48 \mathrm{~h})$ in $5 \mathrm{mM}$ GSH in phosphate-buffered saline (PBS; Supplementary Table 8). These compounds representing different scaffolds were stable under these conditions. Only for the autophagy inhibitor $\mathbf{3 f}$ a slight reactivity was detected after incubation for $48 \mathrm{~h}$. However, the mode of action of $\mathbf{3 f}$ cannot be attributed to this reactivity as the treatment time in the autophagy assay is only $3 \mathrm{~h}$. Therefore, the 
compounds appear to have a non-covalent and reversible mode of target binding/inhibition.

Thus, the compound collection represented by three different scaffolds generated by ligand-directed gold-catalysed rearrangements of 1,6-enynes successfully delivered structurally diverse small molecules selectively active in four different biological assays monitoring major cellular pathways and processes with relevance to human disease.

\section{Discussion}

Organic synthesis has explored only a limited area of chemical space as represented by a small percentage of scaffolds making up the known synthetic small molecules ${ }^{57}$. A similar lack of structural diversity in screening collections remains a major reason for the limited efficiency in translating small-molecule synthesis into pharmaceutical application. Thus, novel synthetic designs are in high demand, in particular concise catalytic synthesis approaches that can be used to efficiently create scaffold diversity in compound collections that are expected to yield highly useful novel small-molecule candidates for drug and probe discovery research. The ligand-directed synthesis strategy described here is a unified synthetic approach, in which common starting materials are exposed to a common mode of metal catalysis leading to a common type of intermediate, whose molecular fate then was steered to yield three structurally different and diverse scaffolds by tuning the metal catalyst through different ligands. Following this approach and using oxindole-derived 1,6-enynes, we successfully established the reaction conditions, wherein different gold(I) complexes steered the substrates to different reaction pathways thereby generating three distinct scaffold classes. Analysis of a compound collection synthesized by means of this ligand-directed synthesis approach for modulation of major cellular signalling pathways and programs revealed structurally diverse, selective and novel modulators of bioactivity. We believe that the unified scaffold diversity synthesis approach that explores the potential of reactive intermediates, whose molecular fate can be selectively steered towards different structures may also be applicable to other modes of catalysis, for instance in palladium ${ }^{6,58}$ and organocatalyzed divergent transformations.

\footnotetext{
Methods

Biological materials. Dulbecco's Modified Eagle's medium (DMEM), L-glutamine, sodium pyruvate, penicillin/streptomycin, fetal bovine serum (FBS) and fetal calf serum (FCS) were obtained from PAN Biotech, Germany. Chemiluminescent substrate CDP-Star was purchased from Roche, Switzerland. Dual-Luciferase Reporter Assay System was obtained from Promega, USA. pGEN mSmo (Addgene no. 37673) was obtained from Addgene, USA. Anti-acetylated tubulin antibody (Sigma-T6793; 1DB-001-0000868584) and anti-Smo antibody (Abcam-ab38686; 1DB-001-0000338068) were purchased from Sigma, Germany and Abcam, UK, respectively. Alexa Fluor 594-conjugated goat anti-rabbit (A11012; 1DB-001-0000889857) and Alexa Fluor 488-conjugated donkey anti-mouse (A21202; 1DB-001-0000889982) antibodies were purchased from Invitrogen, USA.
}

Cell lines. The murine fibroblast cell line NIH/3T3 was obtained from DSMZ, Germany (DSMZ ACC 59) and was cultured in DMEM (high glucose) supplemented with $10 \%$ heat-inactivated FCS, $2 \mathrm{mM}$ L-glutamine and $1 \mathrm{mM}$ sodium pyruvate. Shh Light II cells ${ }^{59}$ (NIH/3T3 cells stably transfected with a Gli-responsive firefly luciferase reporter plasmid and a pRL-TK constituitive Renilla luciferase expression vector) were cultured in the same culturing medium as the parental NIH/3T3 cells supplemented with $400 \mu \mathrm{g} \mathrm{ml}^{-1} \mathrm{G} 418$ and $150 \mu \mathrm{g} \mathrm{ml}^{-1}$ Zeocin as selecting agents. The murine osteoblasts C3H10T1/2 (ATCC CCL-226) were obtained from ATCC, USA and were cultured in DMEM (high glucose) supplemented with $10 \%$ heat-inactivated FCS, $6 \mathrm{mM}$ L-glutamine, $1 \mathrm{mM}$ sodium pyruvate, as well as penicillin and streptomycin. HEK293T cells (ATCC, Middlesex, England) were grown in DMEM containing 10\% FBS, $100 \mathrm{U} \mathrm{ml}^{-1}$ penicillin and $0.1 \mathrm{mg} \mathrm{ml}^{-1}$ streptomycin. All cell lines were maintained at $37^{\circ} \mathrm{C}$ and $5 \% \mathrm{CO}_{2}$ in humidified atmosphere. All the cell lines were regularly assayed for mycoplasma and were confirmed to be mycoplasma-free.
Screening to identify modulators of Hh signalling. For high-throughput screening an osteogenesis assay was performed at the Compound Management and Screen Center (COMAS) in Dortmund, Germany. Briefly, 800 C3H10T1/2 cells were seeded per well in white 384 -well plates. After incubation overnight compounds were added to a final concentration of $10 \mu \mathrm{M}$ using the acoustic nanoliter dispenser ECHO 520 (Labcyte). One hour later, osteogenesis was induced with $1.5 \mu \mathrm{M}$ purmorphamine. The activity of alkaline phosphatase was measured using the chemiluminescent substrate CDP-Star $96 \mathrm{~h}$ after compound addition. One hour after addition of CDP-Star, luminescence was monitored using the Paradigm plate reader (Molecular Devices, USA).

The effect of test compounds on the viability of C3H10T1/2 cells was determined by the CellTiter-Glo Luminescence cell viability assay (Promega). Cells were treated as described for the osteogenesis assay before addition of the CellTiter-Glo reagent and the assay was performed according to manufacturer's protocol. Compounds causing at least a $50 \%$ reduction in the osteogenesis assay and retaining cell viability of at least $80 \%$ were considered as hits. Dose-response analysis was performed for all hit compounds using a threefold dilution series starting from a concentration of $30 \mu \mathrm{M}$. $\mathrm{IC}_{50}$ was calculated using the Quattro software suite (Quattro Research $\mathrm{GmbH}$ ).

Gli-dependent reporter gene assay. Shh Light II cells stably expressing a Gli-responsive firefly luciferase reporter plasmid and a pRL-TK vector for constitutive expression of Renilla luciferase were seeded in 96-well plate $\left(2.5 \times 10^{4}\right.$ cells per well). After overnight incubation, medium was replaced by low-serumcontaining medium ( $0.5 \%$ FCS) supplemented with $1.5 \mu \mathrm{M}$ purmorphamine. One hour later various concentrations of the compounds or dimethyl sulfoxide (DMSO) as a control were added and cells were further incubated for $48 \mathrm{~h}$. Firefly and Renilla luciferase activity were determined by means of the Dual-Luciferase Reporter Assay System (Promega) according to the manufacturer's protocol using the Infinite M200 plate reader (Tecan, Austria).

SAG competition assay. Competition assay was performed using the Glidependent reporter gene assay as described above with exception of using $0.1 \mu \mathrm{M}$ or $1 \mu \mathrm{M}$ Smo agonist (SAG) to activate the pathway instead of purmorphamine

Quantitative PCR. C3H10T1/2 cells were seeded in 24 -well plates $\left(2 \times 10^{4}\right.$ per well). After overnight incubation, cells were treated with $1.5 \mu \mathrm{M}$ purmorphamine and the test compounds or DMSO for $48 \mathrm{~h}$. Complementary DNA was prepared using FastLane Cell cDNA Kit (Qiagen) following the manufacturer's instructions. The expression of Hh target genes Ptch1 and Gli1 and the reference gene Gapdh gene was determined by means of quantitative PCR using the QuantiFast SYBR Green PCR Kit (Qiagen) and iQ 5 Real-Time PCR Detection System (Bio-rad) and the following oligonucleotides: Ptch1: $5^{\prime}$-CTCTGGAGCAGATTTCCAAGG-3' and $5^{\prime}$-TGCCGCAGTTCTTTTGAATG-3', Gli1: 5'-GGAAGTCCTATTCACGCCT TGA- $3^{\prime}$ and $5^{\prime}$-CAACCTTCTTGCTCACACATGTAAG- $3^{\prime}$, Gapdh: $5^{\prime}$-AGCCTCG TCCCG TAGACAAAAT- $3^{\prime}$ and $5^{\prime}$-CCGTGAGTGGAGTCATACTGGA- $3^{\prime 60}$. The expression levels of Ptch 1 and Gli1 were determined using the $2-\Delta \Delta \mathrm{Ct} \operatorname{method}^{61}$.

Smo binding assay using fluorescence microscopy. A total of $1.5 \times 10^{4}$

HEK293T cells were seeded on poly-D-lysine-coated coverslips placed in a 24-well plate. After overnight incubation, cells were transfected with the Smo expressing plasmid pGEN-mSmo (Addgene no. 37673) using Fugene HD (Promega) according to the manufacturer's protocol. After $48 \mathrm{~h}$ incubation at $37^{\circ} \mathrm{C}$, cells were washed twice with PBS and fixed with $3 \%$ paraformaldehyde for $10 \mathrm{~min}$ at room temperature and subsequent permeabilization with $0.2 \%$ sodium azide in $1 \times$ PBS for $5 \mathrm{~min}$ at room temperature. The cells were washed once with PBS and incubated further in fresh DMEM medium containing $0.5 \% \mathrm{FBS}, 5 \mathrm{nM}$ BODIPY-cyclopamine and various concentrations of the test compounds or DMSO as a control. One hour later cells were washed twice with PBS and stained with $1 \mu \mathrm{g} \mathrm{ml}^{-1} 4^{\prime}$,6-diamidino-2-phenylindole for $10 \mathrm{~min}$ and were mounted on glass slides using Aqua Poly/mount (Polysciences Inc). Images were acquired on an Axiovert Observer Z1 microscope (Carl Zeiss, Germany) using a Plan-Apochromat $\times 63 / 1.40$ Oil DIC M27 objective.

Smo binding assay using flow cytometry. A total of $3 \times 10^{5}$ HEK293T cells were seeded per well in six-well plates. After incubation overnight, cells were transfected with the Smo expressing plasmid pGEN-mSmo as described above. Forty-eight hours later medium was replaced by DMEM containing $0.5 \%$ FBS, $5 \mathrm{nM}$ BODIPY-cyclopamine and various concentrations of the test compounds or DMSO as a control. Following incubation for $5 \mathrm{~h}$ cells were washed once with PBS, detached using trypsin/EDTA (0.05/0.02\% in PBS), and collected by centrifugation at $250 \mathrm{~g}$ for $5 \mathrm{~min}$ at room temperature. Cells were washed twice and then suspended in ice-cold PBS. Cell suspensions were subjected to flow cytometry analysis employing the BD LSR II Flow Cytometer (laser line: $488 \mathrm{~nm}$, emission filter: 530/30) to detect the presence of BODIPY. Data analysis was performed using the FlowJo software, version 7.6.5 (Tree Star Inc., USA). 
Ciliary localization of Smo. A total of $3 \times 10^{4} \mathrm{NIH} / 3 \mathrm{~T} 3$ cells were seeded per well in 24-well plates containing coverslips and cultured overnight. Cells were further incubated for $12 \mathrm{~h}$ in DMEM containing 0.5\% FCS to induce ciliation. Cells were then treated with $1.5 \mu \mathrm{M}$ purmorphamine and various concentrations of the test compounds or DMSO as a control. Twelve hours later cells were washed with PBS followed by fixation in $4 \%$ ice-cold paraformaldehyde for $10 \mathrm{~min}$ at room temperature. Permeabilization and blocking of non-specific binding was performed with a solution containing $0.1 \%$ Triton X-100 and $1 \%$ heat-inactivated horse serum in PBS for $30 \mathrm{~min}$ at room temperature. Samples were then incubated with mouse anti- $N$-acetylated tubulin antibody (dilution 1:5,000) and rabbit anti-Smo antibody (dilution 1:200) overnight at $4{ }^{\circ} \mathrm{C}$ followed by washing and subsequent incubation with Alexa Fluor 594-conjugated goat anti-rabbit and Alexa Fluor 488-conjugated donkey anti-mouse antibodies (Invitrogen; 1:500 dilutions) and 4',6-diamidino-2phenylindole $\left(0.1 \mu \mathrm{g} \mathrm{ml}^{-1}\right)$ for $45 \mathrm{~min}$ at room temperature. Coverslips were washed and mounted using Aqua Poly/mount (Polysciences. Inc USA). Images were acquired in a Axiovert Observer microscope Z1 (Carl Zeiss, Germany) using a Plan-Apochromat $\times 63 / 1.40$ Oil DIC M27 objective.

Screening to identify inhibitors of Wnt signalling. The screening was performed at COMAS in Dortmund, Germany. Wnt activity was analysed in HEK293 human embryonic kidney cells stably transfected with the human Frizzled-1 receptor and a luciferase reporter gene under the control of the TOPFLASH motif ${ }^{54}$. Cells were activated by addition of Wnt 3a-conditioned medium that had been collected from $\mathrm{L}$ cells overexpressing the Wnt 3 a protein ${ }^{13}$. Control cells were treated with conditioned medium from L cells. Three thousand HEK293-Fz1-TOPFLASH cells were seeded per well of a 384-well plate and incubated with the compounds for $6 \mathrm{~h}$. XAV939 was used as a control for Wnt pathway inhibition. OneGlo Reagent (Promega Inc.) was added and the luminescence signal was recorded with a Spectramax Paradigm reader (Molecular Devices). As toxic compounds also lead to a decreased luciferase signal, cell viability was assessed in parallel to the Wnt reporter gene assay using the CellTiter-Glo reagent (Promega). Briefly, 1,000 HEK293-Fz1-TOPFLASH cells were seeded per well in a 384-well plate. After a treatment with the compounds for $6 \mathrm{~h}$, the luminescence signal was measured after addition of the CellTiter-Glo reagent. Dose-response analyses were carried out using eight threefold dilution steps starting from $30 \mu \mathrm{M}$. Compounds were dispensed using an Echo520 acoustic dispenser (Labcyte Inc.). $\mathrm{IC}_{50}$ values were calculated with Quattro/Workflow (Quattro research $\mathrm{GmbH}$ ). All measurements were performed in triplicate. To identify compounds that interfere with transcription or translation or directly inhibit luciferase, a HEK293 cell line with constitutive firefly luciferase expression was used. Three thousand cells were seeded per well in a 384-well plate and incubated with the compounds for $6 \mathrm{~h}$. OneGlo Reagent (Promega) was added and the luminescence signal was recorded with a Spectramax Paradigm reader (Molecular Devices). Control cells were treated with $10 \mu \mathrm{M}$ cycloheximide. Dose-response analyses were carried out as described above.

Screening to identify autophagy modulators. Screening for autophagy modulators was performed at COMAS in Dortmund, Germany. Briefly, MCF7-GFP-LC3 (4,000 cells per well) cells were seeded in 384-well plates (Greiner). After incubation overnight cells were washed thrice with PBS using the plate washer ELX405 (Biotek) followed by addition of $10 \mu \mathrm{M}$ of the compounds using the Echo dispenser (Labcyte) along with Earl's Balanced Salt Solution to induced autophagy and chloroquine $(50 \mu \mathrm{M})$. Cells were incubated for $3 \mathrm{~h}$ before fixation using $4.6 \%$ formaldehyde in PBS and staining of the DNA using Hoechst 33342 for $20 \mathrm{~min}$ at room temperature. Fixed cells were washed thrice with PBS using the plate washer ELX405 (Biotek). For visualization, four pictures per well were acquired on an ImageXpress Micro XL (Molecular Devices) at $\times 20$ magnification and analysed with the granularity setting of the MetaXpress Software (Molecular Devices). Wortmannin was used as a control for autophagy inhibition. Dose-response analysis was carried out using eight doses of a threefold dilution series starting at $10 \mu \mathrm{M}$. IC 50 values were calculated using the Quattro Workflow software (Quattro Research $\mathrm{GmbH}$ )

Time-lapse proliferation study in HeLa cells. HeLa cells (3,000 cells per well) were seeded in 96-well clear bottom plates and incubated overnight at $37^{\circ} \mathrm{C}$. On the following day, the medium was replaced by fresh medium containing various concentrations of test compounds and DMSO as a control. Cells were then subjected to live-cell imaging over $96 \mathrm{~h}$ under standard incubation conditions using an IncuCyte ZOOM imaging system (Essen BioScience). Images were acquired every $30 \mathrm{~min}$. Image-based analysis of cell confluence was carried out using the IncuCyte2015A software. All the experiments were performed in triplicates.

GSH reactivity assay. $30 \mu \mathrm{M}$ of the compounds were incubated with PBS or $5 \mathrm{mM}$ GSH for different period of time at $37^{\circ} \mathrm{C}$. Samples were then analysed by high-performance liquid chromatography-mass spectrometry (HPLC: Ultimate 3000, MS: VELOS Pro- Thermo Fisher Scientific, Waltham, MA, USA). Peak areas for the respective molecular weight of the compound incubated with PBS or GSH was employed for comparison. The peak areas were normalized to the respective peak areas in PBS to obtain the per cent abundance of the compounds in GSH.
Chemical synthesis. Compounds were synthesized according to the procedures specified in Supplementary Methods. X-ray crystallographic data and images are reported in Supplementary Tables 3-6. For ${ }^{1} \mathrm{H}$ and ${ }^{13} \mathrm{C}$ nuclear magnetic resonance spectra of compounds see the section of nuclear magnetic resonance spectra in Supplementary Figs 4-134.

Data availability. The X-ray crystallographic coordinates for 2a, 3a, 4a and 14a can be obtained free of charge from The Cambridge Crystallographic Data Centre via www.ccdc.cam.ac.uk/data_request/cif under deposition numbers CCDC $1448677,1448646,1448652$ and 1448645 , respectively. All other data supporting the findings of this study are available within the paper and its Supplementary Information Files.

\section{References}

1. Shelat, A. A. \& Guy, R. K. Scaffold composition and biological relevance of screening libraries. Nat. Chem. Biol. 3, 442-446 (2007).

2. Lee, M. L. \& Schneider, G. Scaffold architecture and pharmacophoric properties of natural products and trade drugs: Application in the design of natural product-based combinatorial libraries. J. Comb. Chem. 3, 284-289 (2001).

3. Garcia-Castro, M. et al. De novo branching cascades for structural and functional diversity in small molecules. Nat. Commun. 6, 6516 (2015).

4. Liu, W., Khedkar, V., Baskar, B., Schürmann, M. \& Kumar, K. Branching Cascades: A Concise Synthetic Strategy Targeting Diverse and Complex Molecular Frameworks. Angew. Chem. Int. Ed. 50, 6900-6905 (2011).

5. Morton, D., Leach, S., Cordier, C., Warriner, S. \& Nelson, A. Synthesis of natural-product-like molecules with over eighty distinct scaffolds. Angew. Chem. Int. Ed. 48, 104-109 (2009).

6. Wang, M., Zhang, X., Zhuang, Y.-X., Xu, Y.-H. \& Loh, T.-P. Pd-Catalyzed intramolecular $\mathrm{c}-\mathrm{n}$ bond cleavage, 1,4-migration, $\mathrm{sp} 3 \mathrm{c}-\mathrm{h}$ activation, and heck reaction: four controllable diverse pathways depending on the judicious choice of the base and ligand. J. Am. Chem. Soc. 137, 1341-1347 (2015).

7. McLeod, M. C. et al. Probing chemical space with alkaloid-inspired libraries. Nat. Chem. 6, 133-140 (2014)

8. Woerly, E. M., Roy, J. \& Burke, M. D. Synthesis of most polyene natural product motifs using just 12 building blocks and one coupling reaction. Nat. Chem. 6, 484-491 (2014)

9. Huigens, R. W. et al. A ring-distortion strategy to construct stereochemically complex and structurally diverse compounds from natural products. Nat. Chem. 5, 195-202 (2013).

10. Balthaser, B. R., Maloney, M. C., Beeler, A. B., Porco, J. A. \& Snyder, J. K. Remodelling of the natural product fumagillol employing a reaction discovery approach. Nat. Chem. 3, 969-973 (2011).

11. Wu, J. et al. Sequential transformations to access polycyclic chemotypes: asymmetric crotylation and metal carbenoid reactions. Angew. Chem. Int. Ed. 50, 5938-5942 (2011).

12. Brown, L. E. et al. Discovery of new antimalarial chemotypes through chemical methodology and library development. Proc. Natl Acad. Sci. USA 108, 6775-6780 (2011).

13. Nieto-Oberhuber, C. et al. Cationic gold(I) complexes: highly alkynophilic catalysts for the exo- and endo-cyclization of enynes. Angew. Chem. Int. Ed. 43, 2402-2406 (2004).

14. Amijs, C. H. M., Ferrer, C. \& Echavarren, A. M. Gold(i)-catalysed arylation of 1,6-enynes: different site reactivity of cyclopropyl gold carbenes. Chem. Commun. 43, 698-700 (2007).

15. Friend, C. M. \& Hashmi, A. S. K. Gold catalysis. Acc. Chem. Res. 47, 729-730 (2014).

16. Jiménez-Núñez, E. \& Echavarren, A. M. Gold-catalyzed cycloisomerizations of enynes: a mechanistic perspective. Chem. Rev. 108, 3326-3350 (2008).

17. Pérez-Galán, P., Martin, N. J. A., Campaña, A. G., Cárdenas, D. J. \& Echavarren, A. M. Carbocations or cyclopropyl gold carbenes in cyclizations of enynes. Chem. Eur. J. 6, 482-486 (2011)

18. Nieto-Oberhuber, C. et al. Gold(I)-catalyzed intramolecular $[4+2]$ cycloadditions of arylalkynes or 1,3-enynes with alkenes: scope and mechanism. J. Am. Chem. Soc. 130, 269-279 (2008).

19. Furstner, A. Gold and platinum catalysis-a convenient tool for generating molecular complexity. Chem. Soc. Rev. 38, 3208-3221 (2009).

20. Chen, G. Q., Fang, W., Wei, Y., Tang, X. Y. \& Shi, M. Divergent reaction pathways in gold-catalyzed cycloisomerization of 1,5 -enynes containing a cyclopropane ring: dramatic ortho substituent and temperature effects. Chem. Sci. 7, 4318-4328 (2016)

21. Ding, D., Mou, T., Feng, M. H. \& Jiang, X. F. Utility of ligand effect in homogenous gold catalysis: enabling regiodivergent pi-bond-activated cyclization. J. Am. Chem. Soc. 138, 5218-5221 (2016).

22. Jiang, Y., Wei, Y., Tang, X. Y. \& Shi, M. Gold(I)-catalyzed selective heterocyclization of propargylic thioureas: mechanistic study of competitive gold-activation mode. Chem. Eur. J. 21, 7675-7681 (2015). 
23. Mei, L. Y., Wei, Y., Tang, X. Y. \& Shi, M. Catalyst-dependent stereodivergent and regioselective synthesis of indole-fused heterocycles through formal cycloadditions of indolyl-allenes. J. Am. Chem. Soc. 137, 8131-8137 (2015).

24. Rao, W. D., Susanti, D., Ayers, B. J. \& Chan, P. W. H. Ligand-controlled product selectivity in gold-catalyzed double cycloisomerization of 1,11-dien-3,9-diyne benzoates. J. Am. Chem. Soc. 137, 6350-6355 (2015).

25. Shiroodi, R. K., Koleda, O. \& Gevorgyan, V. 1,2-Boryl migration empowers regiodivergent synthesis of borylated furans. J. Am. Chem. Soc. 136, 13146-13149 (2014)

26. Wang, Z. X., Wang, Y. Z. \& Zhang, L. M. Soft propargylic deprotonation: designed ligand enables au-catalyzed isomerization of alkynes to 1,3-dienes. J. Am. Chem. Soc. 136, 8887-8890 (2014).

27. Alcarazo, M., Stork, T., Anoop, A., Thiel, W. \& Furstner, A. Steering the surprisingly modular pi-acceptor properties of $\mathrm{n}$-heterocyclic carbenes: implications for gold catalysis. Angew. Chem. Int. Ed. 49, 2542-2546 (2010).

28. Alonso, I. et al. Gold-catalyzed [4C $+2 \mathrm{C}]$ cycloadditions of allenedienes, including an enantioselective version with new phosphoramidite-based catalysts: mechanistic aspects of the divergence between $[4 \mathrm{C}+3 \mathrm{C}]$ and [4C + 2C] pathways. J. Am. Chem. Soc. 131, 13020-13030 (2009).

29. Mauleon, P., Zeldin, R. M., Gonzalez, A. Z. \& Toste, F. D. Ligand-controlled access to $[4+2]$ and $[4+3]$ cycloadditions in gold-catalyzed reactions of allene-dienes. J. Am. Chem. Soc. 131, 6348 (2009).

30. Echavarren, A. M. Carbene or cation? Nat. Chem. 1, 431-433 (2009).

31. Gorin, D. J., Sherry, B. D. \& Toste, F. D. Ligand effects in homogeneous Au catalysis. Chem. Rev. 108, 3351-3378 (2008).

32. Obradors, C. \& Echavarren, A. M. Intriguing mechanistic labyrinths in gold(I) catalysis. Chem. Commun. 50, 16-28 (2014).

33. Wittstein, K., Kumar, K. \& Waldmann, H. Gold(I)-Catalyzed synthesis of benzoxocines by an 8-endo-dig cyclization. Angew. Chem. Int. Ed. 50, 9076-9080 (2011).

34. Kolundžić, F. et al. A cyclization-rearrangement cascade for the synthesis of structurally complex chiral gold(i)-aminocarbene complexes. Angew. Chem. Int. Ed. 53, 8122-8126 (2014).

35. Galliford, C. V. \& Scheidt, K. A. Pyrrolidinyl-spirooxindole natural products as inspirations for the development of potential therapeutic agents. Angew. Chem. Int. Ed. 46, 8748-8758 (2007).

36. Ding, K. et al. Structure-based design of spiro-oxindoles as potent, specific small-molecule inhibitors of the MDM2 - p53 interaction. J. Med. Chem. 49, 3432-3435 (2006).

37. Antonchick, A. P. et al. Highly enantioselective synthesis and cellular evaluation of spirooxindoles inspired by natural products. Nat. Chem. 2, 735-740 (2010).

38. Cao, Y. M. et al. Enantioselective Michael/Cyclization Reaction Sequence: Scaffold-Inspired Synthesis of Spirooxindoles with Multiple Stereocenters. Angew. Chem. Int. Ed. 50, 9124-9127 (2011).

39. Spada, L. T. et al. Kinase inhibitors (Google Patents, 2011).

40. Peng, P., Tang, B.-X., Pi, S.-F., Liang, Y. \& Li, J.-H. Synthesis of (E)-3(isobenzofuran-3(1H)-ylidene)indolin-2-ones by the palladium-catalyzed intramolecular $\mathrm{C}-\mathrm{H}$ functionalization process. J. Org. Chem. 74, 3569-3572 (2009).

41. Imbulgoda, A., Heng, D. C. \& Kollmannsberger, C. in Small Molecules in Oncology, Vol. 201 (eds Martens, U. M. et al.) 165-184 (Springer Berlin Heidelberg, 2014).

42. Jiang, X. X. et al. A unique approach to the concise synthesis of highly optically active spirooxazolines and the discovery of a more potent oxindole-type phytoalexin analogue. J. Am. Chem. Soc. 132, 15328-15333 (2010).

43. Lin, T. T. et al. Prenylated coumarins: natural phosphodiesterase- 4 inhibitors from Toddalia asiatica. J. Nat. Prod. 77, 955-962 (2014).

44. Ferrer, C., Raducan, M., Nevado, C., Claverie, C. K. \& Echavarren, A. M. Missing cyclization pathways and new rearrangements unveiled in the gold(I) and platinum(II)-catalyzed cyclization of 1,6-enynes. Tetrahedron $\mathbf{6 3}$, 6306-6316 (2007).

45. Soriano, E. \& Marco-Contelles, J. Mechanistic insights on the cycloisomerization of polyunsaturated precursors catalyzed by platinum and gold complexes. Acc. Chem. Res. 42, 1026-1036 (2009).

46. Obradors, C. \& Echavarren, A. M. Gold-catalyzed rearrangements and beyond. Acc. Chem. Res. 47, 902-912 (2014).

47. Amijs, C. H. M. et al. Gold(I)-catalyzed intermolecular addition of carbon nucleophiles to 1,5- and 1,6-enynes. J. Org. Chem. 73, 7721-7730 (2008).

48. Briscoe, J. \& Therond, P. P. The mechanisms of Hedgehog signalling and its roles in development and disease. Nat. Rev. Mol. Cell. Biol. 14, 416-429 (2013).
49. Wu, X., Walker, J., Zhang, J., Ding, S. \& Schultz, P. G. Purmorphamine induces osteogenesis by activation of the hedgehog signaling pathway. Chem. Biol. 11, 1229-1238 (2004).

50. Taipale, J. et al. Effects of oncogenic mutations in Smoothened and Patched can be reversed by cyclopamine. Nature 406, 1005-1009 (2000).

51. Chen, J. K., Taipale, J., Young, K. E., Maiti, T. \& Beachy, P. A. Small molecule modulation of Smoothened activity. Proc. Natl Acad. Sci. USA 99, 14071-14076 (2002).

52. Sharpe, H. J., Wang, W., Hannoush, R. N. \& de Sauvage, F. J. Regulation of the oncoprotein Smoothened by small molecules. Nat. Chem. Biol. 11, 246-255 (2015).

53. Anastas, J. N. \& Moon, R. T. WNT signalling pathways as therapeutic targets in cancer. Nat. Rev. Cancer. 13, 11-26 (2013).

54. Park, S. et al. Hexachlorophene inhibits Wnt/beta-catenin pathway by promoting Siah-mediated beta-catenin degradation. Mol. Pharmacol. 70, 960-966 (2006).

55. Kaur, J. \& Debnath, J. Autophagy at the crossroads of catabolism and anabolism. Nat. Rev. Mol. Cell. Biol. 16, 461-472 (2015).

56. Choi, K. S. Autophagy and cancer. Exp. Mol. Med. 44, 109-120 (2012).

57. Lipkus, A. H. et al. Structural diversity of organic chemistry. A scaffold analysis of the CAS Registry. J. Org. Chem. 73, 4443-4451 (2008).

58. Kim, J. H., Bouffard, J. \& Lee, S. G. Formation of four different aromatic scaffolds from nitriles through tandem divergent catalysis. Angew. Chem. Int. Ed. 53, 6435-6438 (2014).

59. Chen, J., Wang, W. \& Liu, Z. X. Transient anti-plane crack problem for a functionally graded piezoelectric coating bonded to an elastic layer. Key Eng. Mat. 274-276, 1119-1124 (2004).

60. Lipinski, R. J., Gipp, J. J., Zhang, J., Doles, J. D. \& Bushman, W. Unique and complimentary activities of the Gli transcription factors in Hedgehog signaling. Exp. Cell Res. 312, 1925-1938 (2006).

61. Livak, K. J. \& Schmittgen, T. D. Analysis of relative gene expression data using realtime quantitative PCR and the $2-\Delta \Delta$ CT method. Methods 25, 402-408 (2001).

\section{Acknowledgements}

The research was supported by the Max Planck Gesellschaft and the European Research Council under the European Union's Seventh Framework Programme (FP7/2007-2013)/ ERC Grant agreement no. 268309. The Compound Management and Screening Center (COMAS, Dortmund) is acknowledged for carrying out the screening and data analysis. Y.-C.L. like to thank Ministry of Education (Taiwan) for the MOE Technologies Incubation Scholarship.

\section{Author contributions}

Y.-C.L. performed chemical experiments. S.P. performed biological experiments. C.G. and C.S. determined crystal structure analyses. S.Z., K.K. and H.W. supervised the research. Y.-C.L., S.P., S.Z., K.K. and H.W. designed experiments and wrote the manuscript.

\section{Additional information}

Supplementary Information accompanies this paper at http://www.nature.com/ naturecommunications

Competing financial interests: The authors declare no competing financial interests

Reprints and permission information is available online at http://npg.nature.com/ reprintsandpermissions/

How to cite this article: Lee, Y.-C. et al. A ligand-directed divergent catalytic approach to establish structural and functional scaffold diversity. Nat. Commun. 8, 14043 doi: $10.1038 /$ ncomms14043 (2017).

Publisher's note: Springer Nature remains neutral with regard to jurisdictional claims in published maps and institutional affiliations.

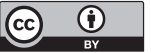

This work is licensed under a Creative Commons Attribution 4.0 International License. The images or other third party material in this article are included in the article's Creative Commons license, unless indicated otherwise in the credit line; if the material is not included under the Creative Commons license, users will need to obtain permission from the license holder to reproduce the material. To view a copy of this license, visit http://creativecommons.org/licenses/by/4.0/

(C) The Author(s) 2017 